\title{
Densitometric analysis of Radiation Absorbed Dose to Patients Undergoing Chest X-ray Examination in a Government Owned Hospital, Kano-Nigeria.
}

\author{
N. F Isa ${ }^{1,2}$, Ahmad U. F. ${ }^{1}$, Dambele $M Y^{3}$, \\ Ibrahim U.M. ${ }^{2}$, A. Ismail ${ }^{4}$, Taofeeq A. Ige ${ }^{5}$ \\ ${ }^{1}$ Centre for Renewable Energy Research, \\ Bayero University, \\ Kano, Nigeria. \\ ${ }^{2}$ Department of Physics, \\ Bayero University, \\ Kano, Nigeria. \\ ${ }^{3}$ Department of Medical Radiography, \\ Bayero University, \\ Kano, Nigeria. \\ ${ }^{4}$ Department of Radiology, \\ Bayero University, \\ Kano, Nigeria. \\ ${ }^{5}$ National Hospital \\ Abuja, Nigeria.
}

Email: ufahmad.crer@buk.edu.ng

\begin{abstract}
Chest X-ray is one the most frequently required diagnostic procedures used in clinical practice to produce an image of organs, tissues and bones of the body with a significant impact on collective dose. However, the radiation dose received by patients from this procedure is likely to show large variations within and among radiodiagnostic centres. This could be traced to be lack of imaging protocols and a lack of local or national diagnostic reference levels. Although the radiation doses resulting from the Xray procedures are usually measured in terms of effective dose entrance surface dose using electronic personal dosimeter (EPD), pocket dosimeter, thermoluminescent dosimeter (TLD), film badges, and optically stimulated luminescent dosimeter (OSLD). For the sake of radiation protection, densitometric analysis was used to evaluate medical exposure for chest radiographs to determine the radiation doses using a point optical densitometer; PTW-DensiX with a range of 0 to 4.0 optical density. The hospital where this study was carried out is in Kano State. A total of sixty (60) chest radiographic films were collected from the hospital and the optical density of each radiographic film was measured five times at different spots. The mean optical density was obtained and converted by a mathematical relation to absorbed radiation dose in $c G y$. The results obtained in this study show that the mean absorbed X-ray dose was $0.78 \pm 0.13 \mathrm{cGy}$ and is low compared to the previous study held for
\end{abstract}

*Author for Correspondence 
hospitals in Osun State. Also, when compared with the excess dose of 0.10 Gy recommended by the International Commission on Radiological Protection, it was evident that radiological practice especially as it relates to chest radiographs at the hospital in Kano is within the recommended practice.

Keywords: Absorbed dose, Densitometer, Optical Density, Radiation, X-ray

\section{INTRODUCTION}

Medical applications of ionizing radiation are considered to be the second source of exposure after natural sources of radiation exposure to man (Streffer, 2007). This largely comes from medical X-ray procedures to patients in diagnosis and therapy. Therefore, dose optimization to patients is necessary to keep it as low as reasonably achievable (ALARA) (European Parliament, 2014). The annual effective dose has increased from medical imagining examinations, where absorbed radiation dose in an organ is usually used for estimating radiation risks to patients (UNSCEAR, 2011). For the past century, low cost of $x-$ ray imaging has become the daily common diagnostic procedure because it has the potential to reduce the patient dose (Veldkamp, Kroft and Geleijns, 2009) in addition when focused on the chest, it can help spot the abnormalities or diseases of the airways, blood vessels, bones, heart and lungs. This makes it have more advantages for diagnosing a variety of health issues (Brian Krans, 2020). According to the ICRP, any medical imaging procedure must be justified based on benefit to the patient. Furthermore, there should be no practice involving exposure to ionizing radiation lest it produces necessary benefit to the exposed individual (Thomas et al., 2005).

Nigeria is a country that has shown an increase in the use of X-ray machines for medical diagnosis with approximately 5,000 machines distributed across the country in both private and public diagnostic centres (Idowu and Okedere, 2020). Although it is difficult to estimate the number of machines that are currently functioning, this increased hence an increasing concern for potentially high radiation dose in X-ray examination as against the guided guideline by international procedures to monitor dose exposure to patience (Koenig, Mettler and Wagner, 2001). Information about radiation absorbed dose is calibrated in some recently designed X-ray machines. Such a device consists of an ionization chamber that permits radiation input to be entered directly. For machines lacking this design, (especially in developing countries) alternative method to measure radiation output is needed to protect patient high exposure (Thomas, 2003). This method was previously investigated by placing a radiographic film at the output of an X-ray machine and then using a densitometer to measure the film response. Therefore, Radiographic film can record high radiation doses by recording the skin entrance radiation dose (Thomas et al., 2005). An optical densitometer was also used with simple fabricated equipment at the Federal Medical Centre, Keffi, Nasarawa State, to determine radiological parameters such as dose, beam alignment, peak kilovoltage and tube current consistency (Goriya et al., 2015). In another study conducted by Osahon et al, (2017) on chest radiographs using densitometer, the Mean Optical Density (MOD) was determined and a mathematical relation was used to convert the MOD to absorbed radiation dose in centi Gray (cGy) (Osahon et al, 2017).

A densitometer is a device used to numerically determine the amount of blackness on the radiograph (i.e., it measures radiographic density). This device is constructed to emit a constant intensity of light (incident) onto an area of the image and then measure the amount of light transmitted through the area. The densitometer determines the amount of light transmitted and calculates the Optical Density (D) (Johnston, Fauber and others et al, 2015). 
In this study, for the first time in North-West Nigeria, we follow up a pilot study by Osahon et al (2017) where absorbed radiation doses from chest X-ray examination were calculated from mean optical density (MOD) using a recognized equation. The result was compared with previous studies to establish the recommendation by the International Commission on Radiological Protection (ICRP).

\section{METHODOLOGY}

This study was conducted using 60 chest radiographs obtained from the a tertiary hospital in Kano, North-Western Nigeria. Chest radiographs of adult patients (18yrs and above) with a weight of $70 \pm 15 \mathrm{~kg}$ diagnosed with heart conditions were considered. Furthermore, patient medical history, where possible were used in this study to compare with the radiology results obtained. Over penetrated radiographs were not considered in this study.

The following abbreviations were adopted for this study:

Optical Density: D

Absorbed X-ray Dose: X

Mean Optical Density: $\mathrm{D}_{M O D}$

The optical density (D) where the heart appeared on the of radiographic films (each) was measured repeatedly five times at different spots on each film as optical densities D1, D2, D3, D4 and D5 using a point optical densitometer, model PTW-DensiX with a range of 0 to 4.0 optical density. The mean of the five optical densities for each film was taken to obtain the mean optical densities $\left(\mathrm{D}_{M O D}\right)$, i.e.

$$
D_{M O D}=\frac{D 1+D 2+D 3+D 4+D 5}{5}
$$

This was repeated for the radiographs and the optical densities were converted to the amount of X-ray radiation dose absorbed by each patient's heart (absorbed X-radiation dose) denoted by $X$. The various standard deviation (SD) was also estimated for each Mean Optical Densities (MOD) of the radiographic film and the absorbed X-ray radiation dose X. The blackening of the film after $\mathrm{X}$-ray radiation exposure is expressed in terms of its optical density as:

$$
D=\log _{10}\left(\frac{I_{0}}{I}\right)
$$

The optical density $\mathrm{D}$ and the maximum number of sensitized grains have a correlation that results in a relation between the optical density D and the absorbed dose $X$, as (Artur, 2003)

where $D_{\text {max }}=4$ and $k=9.36$ [12].

$$
D_{M O D}=D_{\max }\left[1-e^{-k X}\right]
$$

Therefore equation (3) becomes,

$$
D_{M O D}=4\left[1-e^{-9.36 X}\right]
$$

Solving equation (4) for the absorbed $X$-ray radiation dose $X$, gives,

$$
X=\left(-\frac{1}{9.36}\right) \log _{e}\left(1-\frac{D_{M O D}}{4}\right)
$$

Equation (5) was developed into a densitometric simulator to perform the task of calculating the amount of the absorbed X-ray dose $X(\mathrm{cGy})$ for each radiographic film from the MOD. The software runs in Python 3.6 IDLE.

The standard deviation (SD), $\sigma$, of each of the measured optical densities (D) was estimated from the relation:

where:

$$
\sigma=\sqrt{\left(\frac{1}{N} \sum_{i=1}^{N}\left(D_{i}-D_{M O D}\right)\right)^{2}}
$$

$\mathrm{N}$ : is the total number of times the optical densities were measured per radiographic film.

$\mathrm{D}_{\mathrm{i}}$ : is the optical density of each radiographic film.

$\mathrm{D}_{\mathrm{MOD}}$ : is the Mean Optical Density (MOD) of the radiographic film. 


\section{RESULTS AND DISCUSSION}

The results from the exposure parameters are summarized in Table 1.

Table 1. Exposure parameters and statistics of the estimated radiation absorbed dose.

\begin{tabular}{|l|l|}
\hline Parameters & Value \\
\hline $\mathrm{kVp}(\mathrm{min} / \mathrm{max})$ & $45 / 85$ \\
\hline $\mathrm{mAs}(\mathrm{min} / \mathrm{max})$ & $0.5 / 6$ \\
\hline MOD & 0.28 \\
\hline Estimated Absorbed Radiation (cGy) & \\
\hline Min & 0.58 \\
\hline Max & 0.95 \\
\hline Mean & 0.78 \\
\hline Range & 0.37 \\
\hline SD & 0.13 \\
\hline Total number of films & 60 \\
\hline
\end{tabular}

Table 1 shows the statistics of the estimated absorbed dose and the exposure parameters of the $x$-ray machine. The values of absorbed radiation dose, X (cGy), vary from this study compared to the previous literature as confirmed by Osahon et al in Fig 1. These differences cannot be attributed to only one factor but rather factors such as patient size, film screen speed, filtration and technical parameters. Presently in the region Nigeria, there is no known Established Diagnostic Reference Level for this purpose both locally and corresponding national value nationally to compare this study., and that-Nonetheless, NDRL is not universal but specific to a country, personnel and equipment (Olowookere et al., 2012). This study shows that at minimum exposure, $x$-ray examinations can provide the best benefit to the exposed individual. Therefore, further studies are encouraged to add to this library (present study and Osahon et al) so as to establish the anatomical and clinical DRL both locally and nationally.

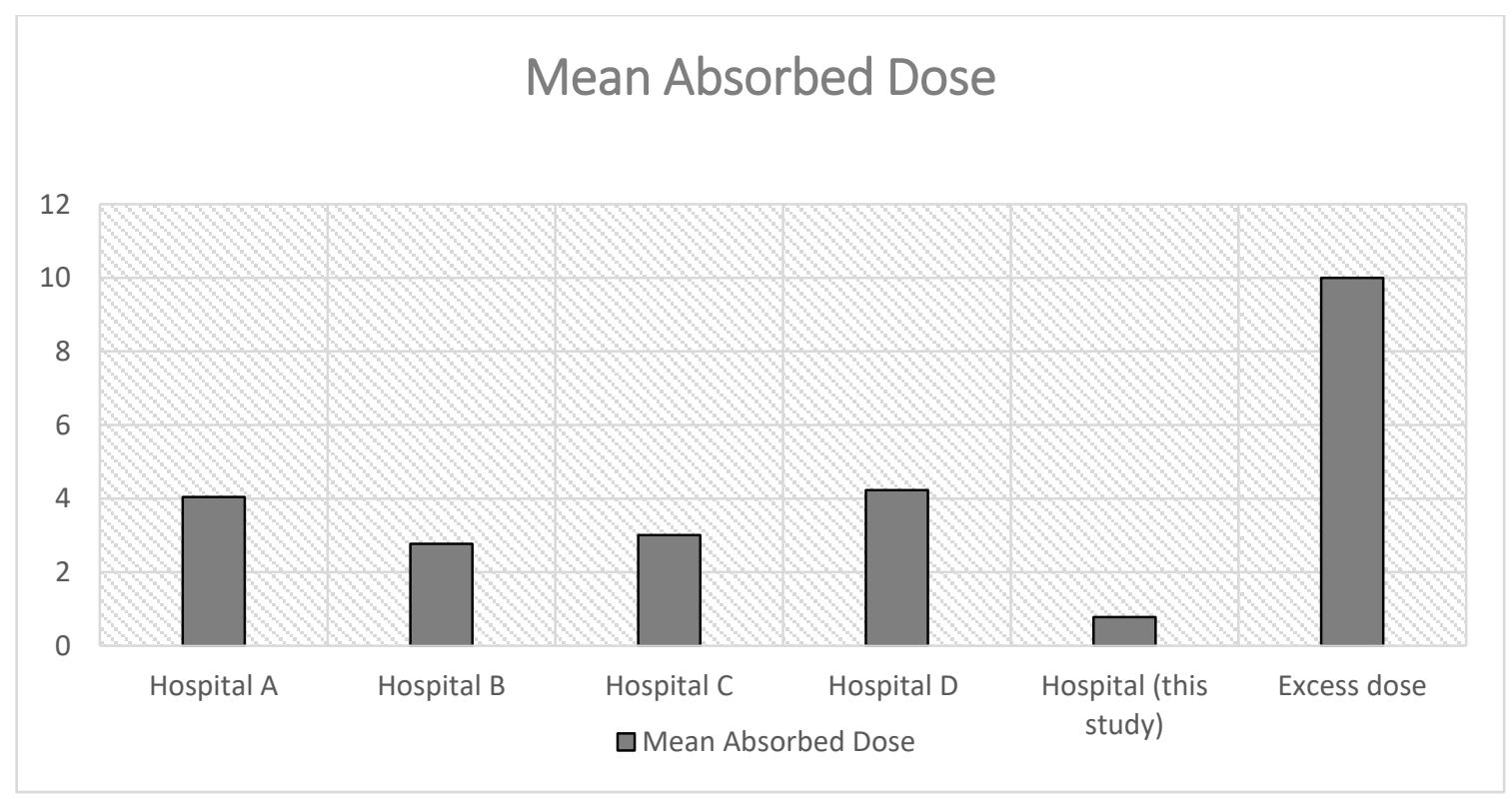

Figure 1. Comparison of mean absorbed dose with previous studies. 


\section{CONCLUSION}

For Quality Assurance (QA) and Quality Control, this study can be adopted to guide against excessive radiation dose to patients during an chest X-ray examination. Also, it was evident that radiological practice especially as it relates to chest radiographs in Kano, Nigeria is within the recommended practice.

\section{ACKNOWLEDGEMENT}

We deeply thank the anonymous reviewers for their thoughtful suggestions that resulted in a much-improved revision of this paper. We would like to thank Prof. T.H. Darma of the Physics Department, Bayero University, Kano, for his guidance throughout this work.

\section{REFERENCES}

Artur, T. (2003) 'Determination of Absorbed X-ray Radiation Dose in X-ray Diagnostics and Imaging', J. Med. Sci, 1(2), pp. 31-36.

Brian Krans (2020) X-Ray: Purpose, Procedure, and Risks. Available at: https://www.healthline.com/health/x-ray.

European Parliament (2014) ‘Council Directive 2013/59/Euratom of 5 December 2013 laying down basic safety standards for protection against the dangers arising from exposure to ionising radiation, and repealing Directives 89/618/Euratom, 90/641/Euratom, 96/29/Euratom, 97/43/Euratom a', Off J Eur Commun L13, (December 2003), pp. 173.

Goriya, K. et al. (2015) 'Determination of Radiological Quality Parameters Using Optical Densitometer Determination of Radiological Quality Parameters Using Optical Densitometer and Simple Fabricated Equipment', (February), pp. 10-15. doi: 10.9790/4861-07121115.

Idowu, B. M. and Okedere, T. A. (2020) 'Diagnostic Radiology in Nigeria: A Country Report', Journal of Global Radiology, 6(1). doi: 10.7191/jgr.2020.1072.

Johnston, J., Fauber, T. L. and others (2015) Essentials of radiographic physics and imaging. Elsevier Health Sciences.

Koenig, T. R., Mettler, F. A. and Wagner, L. K. (2001) 'Skin injuries from fluoroscopically guided procedures: Part 2, review of 73 cases and recommendations for minimizing dose delivered to patient', American Journal of Roentgenology, 177(1), pp. 13-20. doi: 10.2214/ajr.177.1.1770013.

Olowookere, C. J. et al. (2012) 'A Preliminary Radiation Dose Audit in some Nigerian Hospitals: Need for Determination of National Diagnostic Reference Levels (NDRLs)', The Pacific Journal of Science and Technology, 487(1), pp. 487-495.

Osahon, O. D., Ojo, O. A. and Ushie, P. O. (2017) 'Estimation of Radiation Absorbed Dose to Patients Undergoing Chest X-ray Examination in Four Government Own Hospitals in Nigeria', International Journal of Biophysics, 7(2), pp. 24-32. doi: 10.5923/j.biophysics.20170702.02.

Streffer, C. (2007) 'The ICRP 2007 recommendations', Radiation Protection Dosimetry, 127(1-4), pp. 2-7. doi: $10.1093 / \mathrm{rpd} / \mathrm{ncm} 246$.

Thomas, G. (2003) 'A study of GafChromic XR Type R film response with reflective-type densitometers and economical flatbed scanners', Journal of Applied Clinical Medical Physics, 4(4), p. 307. doi: 10.1120/1.1621373.

Thomas, G. et al. (2005) 'Measurement of dose-area product with GafChromic XR Type R film.', Journal of applied clinical medical physics / American College of Medical Physics, 6(3), pp. 122-132. doi: 10.1120/jacmp.v6i3.2047. 
UNSCEAR (2011) Scientific Report: summary of low-dose radiation effects on health, Report of the United Nations Scientific Committee on the Effects of Atomic Radiation 2010. Available at: http://www.unscear.org/docs/publications/2010/UNSCEAR_2010_Report.pdf.

Veldkamp, W. J. H., Kroft, L. J. M. and Geleijns, J. (2009) ‘Dose and perceived image quality in chest radiography', European Journal of Radiology, 72(2), pp. 209-217. doi: 10.1016/j.ejrad.2009.05.039. 